\title{
Lattice determination of the Kaon form factors from ETMC
}

\author{
Federico Mescia* ${ }^{* \dagger}$ \\ Dep. ECM and ICC, Universitat de Barcelona, Spain \\ E-mail: mescia@ub. edu
}

In the last few years, the European Twisted Mass Collaboration (ETMC) has been investigating (maximally) twisted-mass fermions for dynamical QCD simulations and reliable simulations has been carried out for very fine lattice spacings $(a=0.07 \mathrm{fm})$ and for pion masses as light as $260 \mathrm{MeV}$.

Maximally twisted-mass fermions, like Ginsparg-Wilson ones, are based from first principle QCD action, and have the necessary features to guarantee both $O\left(a^{2}\right)$ improvement and simple renormalisation patterns. Moreover, with respect to Ginsparg-Wilson fermions, they have the advantage to be computationally cheap.

Twisted-mass simulations have already been applied by ETMC to calculate several physical quantities in the light, strange and beauty sector. Here, I will only concentrate on Kaon physics results.

2009 KAON International Conference

June 09 - 12, 2009

Tsukuba, Japan

*Speaker.

${ }^{\dagger}$ We thank all the ETMC members for fruitful discussions and in particular P. Dimopoulos and V. Gimenez. Work supported by CUR Generalitat de Catalunya under project 2009SGR502 and by the Consolider-Ingenio 2010 Program CPAN (CSD2007-00042). 


\section{Introduction}

I will review the lattice estimates of the European Twisted Mass Collaboration (ETMC) o Kaon Physics. ETMC [1] is a collaboration of European lattice research centres that exploits maximally twisted Wilson fermions [2,3] for dynamical QCD simulations.

Current ETMC investigations mostly involve partially quenched simulations with two dynamical degenerate (sea) quarks ( $N_{F}=2$ setup), whereas the production of configurations including also dynamical strange and charm quarks $\left(N_{F}=2+1+1\right.$ setup) is in progress [4]. This will allow us to remove the systematic error of quenching the strange quark in the $N_{F}=2$ setup. The merit of present $N_{F}=2$ ETMC studies has however to prove that twisted-mass fermions at maxiaml twist are a powerful approach to predict physics quantities. Besides in these studies, very light dynamical (sea) quark masses have been reached at rather large physical volume: our unitarity pions are as light as $260 \mathrm{MeV}$ at $L m_{\pi} \gtrsim 4$.

In this write-up I will focus on the hadronic uncertainties, $f_{+}(0), f_{K} / f_{\pi}$ and $B_{K}$, of the following Kaon observables:

- $K \rightarrow \pi \ell v$ decays: $B r\left(K_{\ell 3}\right) \propto f_{+}^{2}(0) \times\left|V_{u s}\right|^{2}$.

Here $f_{+}(0)$ is needed to estimate the Cabibbo-Kobayashi-Maskawa (CKM) $V_{u s}$ from the experimental branching ratios.

Thanks to the new measurements of $B r\left(K_{\ell 3}\right)$ by BNL-E865, KLOE, KTeV, ISTRA+, and NA48, the experimental accuracy on $\left|V_{u s}\right| f_{+}(0)$ is about $0.2 \%$ [5]. To translate such a highly precise measurement to a similar error on the $\left|V_{u s}\right|$ determination, the obstacle is to keep the theoretical uncertainties in $f_{+}(0)$ at the per-mil level. Although this target looks quite hard, recent lattice developments are very promising.

From our $N_{F}=2$ twisted-mass calculation [6], we obtain $f_{+}(0)=0.9560(57)_{\text {stat. }}(62)_{\text {syst. }}$ in good agreement with recent both $N_{f}=2$ and $N_{f}=2+1$ lattice determinations (see fig. 4 in sec. 4 ). Although the current overall uncertainty is still at percent level, room of improvement within twisted-mass fermions looks still possible. Now, our study is related to a single lattice spacing and to $N_{F}=2$ fermions. Discretisation and finite volume errors can be reduced by the larger simulations, which are currently in progress. Moreover, half of the systematic error in the value above of $f_{+}(0)$, corresponds to a conservative estimate of the effect of quenching the strange.

Lett's remind that current lattice estimates of $f_{+}(0)$ already allows us to make an important test $[5,7]$ of Standard Model (SM) through the CKM relation $\left|V_{u d}\right|^{2}+\left|V_{u s}\right|^{2}+\left|V_{u b}\right|^{2}=1$. - $K \rightarrow \mu v: B r\left(K_{\ell 2}\right) \propto m_{\ell}^{2} f_{K}^{2} \times\left|V_{u s}\right|^{2}$.

A complementary research to estimate $\left|V_{u s}\right|$ is made through the $K_{\ell 2}$ decays. To minimize the hadronic uncertainties from $f_{K}$, one introduces the ratio $B r\left(K_{\ell 2}\right) / B r\left(\pi_{\ell 2}\right) \propto f_{K}^{2} / f_{\pi}^{2}\left|V_{u s}\right|^{2} /\left|V_{u d}\right|^{2}$, being the $S U$ (3) factor, $f_{K} / f_{\pi}$ better determined on the lattice. Thanks to the KLOE measurement of $K^{+} \rightarrow \mu^{+} v$, the experimental precision of $f_{K} / f_{\pi}\left|V_{u s}\right| /\left|V_{u d}\right|$ [5] is $0.2 \%$. To match such a precision by lattice calculations, we have still to wait for some time. However, thanks to recent lattice development, a big step forward has been already done and the present $f_{K} / f_{\pi}$ estimates are quite reliable. The relative accuracy is at $\mathscr{O}(1 \%)$. In our paper [9], we show that twisted-mass fermions are a valuable approach to precisely measure $f_{K} / f_{\pi}$. Our value is $f_{K} / f_{\pi}=1.210(6)_{\text {stat. }}(17)_{\text {syst. }}$ and turns out to be consistent with recent other lattice determinations (see fig. 3 in sec. 3). Uncertainties are dominated by systematics which are going to be reduced. In particular, we have conservatively 
assumed a relative systematic error of $1.3 \%$ from residual discretisation and finite volume effects to our final results for $f_{K}$ and $f_{K} / f_{\pi}$.

It is worth to remind that a possible deviation of $\left|V_{u s}\right|_{K_{\ell 3}} /\left|V_{u s}\right|_{K_{\ell 2}}$ from unity represents exciting avenue for searches of physics beyond SM $[8,10]$.

- $K^{0}-\bar{K}^{0}$ oscillation: $\varepsilon_{K} \propto \kappa_{\text {eps }} B_{K} \times f(\bar{\rho}, \bar{\eta})$

$\varepsilon_{K}$ is the parameter controlling CP-violation effects in the $K^{0}-\bar{K}^{0}$ oscillations. $\bar{\rho}$ and $\bar{\eta}$ are CKM couplings, parametrising the apex of the unitary triangle and $\kappa_{e p s}=0.92(2)$ ref.[11] stands for sub-leading long-distance QCD effects.

Being $\varepsilon_{K}$ loop suppressed in the SM, it is quite suited for New Physics searches. Recently, a tension of $2.2 \sigma$ between the SM value of $\varepsilon_{K}$ and its experimental value has emerged [12, 11]. To finally clarify this tension, reliable estimates of $B_{K}$ by lattice QCD are needed. From our preliminary study, we obtain for $B_{K}^{R G I}=0.73(3)_{\text {stat. }}(3)_{\text {syst. }}$. From fig. 5 in sec. 5), we see that this value is in good agreement with recent $N_{F}=2+1$ estimates.

In what follows, I will first introduce the recent lattice developtments, next the twisted-mass QCD formalism, and finally I will describe in details our ETMC determinations.

\section{Recent Lattice Developments and Twisted-Mass QCD Fermions}

For long time, lattice simulations have worked in the "so-called" quenched approximations, where sea quark loops are neglected and pion masses were as heavy as $500 \mathrm{MeV}$. This era is now over and many collaborations have performed (reliable) unquenched simulations with $m_{\pi} \lesssim$ $250 \mathrm{MeV}$. Moreover, thanks to recent technical and conceptual developments [20], most simulations are now carried out with first-principle lattice QCD formulation such as Domain Wall (RBC/UKQCD [13]), Wilson-like (BMW [14], CERN-ToV/CLS [15], ETMC [1], QCDSF [16], and PACS-CS [17]) and overlap fermions (JLQCD/TWQCD [18]). This allows us to remove, in addition to quenched uncertainty, the one coming from using staggered fermiosn for dynamical simulations. Staggered approach has been very effective to assess first unquenched results but it relies on an ansatz (the so-called fourth root trick) which hide unknown uncertainties.

Among the new lattice simualtion, twisted-mass fermions offer a comparatively simple framework where useful properties (like automatic $\mathscr{O}\left(a^{2}\right)$ improvement and simple renormalisation pattern) of Domain-Wall and Overlap fermions are guaranteed at a cheap computational scheme. Twisted-mass fermions are Wilson-Like fermions characterized by a rotated Wilson term with respect to the mass term ${ }^{1}$,

$$
S_{\mathrm{F}}=a^{4} \sum_{x} \bar{\psi}_{f}(x)\left[\nabla+a r_{f} e^{-i \omega \gamma_{5}} W_{\mathrm{cr}}+m_{q}\right] \psi_{f}(x) .
$$

Maximally-twisted-mass fermions used by ETMC are for $\omega=\pi / 2$ and for sea $\left(N_{F}=2\right.$ or $N_{F}=$ $2+1+1)$ quarks organized in doublets of opposite $\mathrm{r}$-Wilson parameters $\left(r_{U}=-r_{D}=1\right)$.

The first merit of a rotated wilson term in $S_{\mathrm{F}}$ is that the associated determinant is positive definite for any $\omega \neq 0$. This gives protection against unphysical small eigenvalues and leads to stable and cheap simulations for light pions. The only drawback of twisted mass fermions is the

\footnotetext{
${ }^{1}$ Lett's remind that the condition $\omega=0$ and $r_{f}=1$ for all the flavours in eq. (2.1) represents the standard Wilson fermions, which is the most used lattice regularisation in the quenched era.
} 


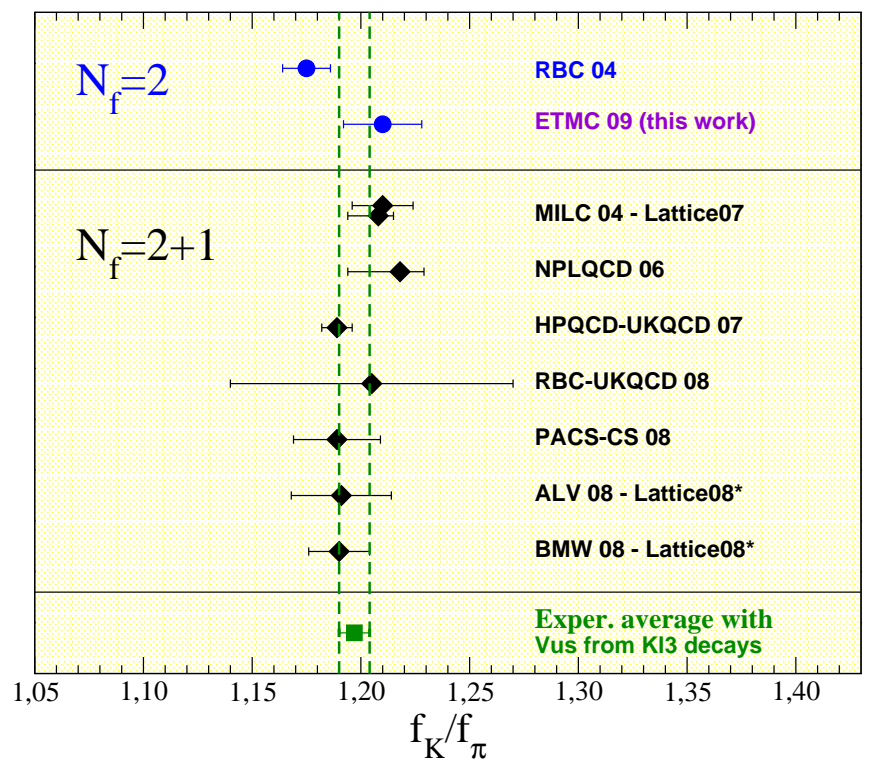

Figure 1: Lattice QCD determinations of the ratio $f_{K} / f_{\pi}$ obtained from simulations with $N_{f}=2$ [22] and $N_{f}=2+1[13,14]$, [23]-[26] dynamical quarks. The results are also compared with the experimental average of $f_{K} / f_{\pi}$ obtained by using for $V_{u s}$ the determination from $K_{\ell 3}$ decays [5].

explicit breaking of parity and isospin by $W_{\text {cr }}$. These breakings are at maximal twist angle $\mathscr{O}\left(a^{2}\right)$ artifacts, and recent ETMC study [21] shows that are under control in the observables entering this write-up.

\section{ETMC determinations of $f_{K}$ and $f_{\pi}$}

The use of twisted-mass fermions at maximal twist turns out to be highly beneficial for pseudoscalar decay constants, $f_{P} \propto\left\langle 0 \bar{q}_{1} \gamma^{\mu} q_{2} \mid P\right\rangle$, since they are automatically improved at $\mathscr{O}(a)$ [3], and their determination through the PCAC identity does not require the introduction of any renormalisation constant. Both these features (typical of chiral invariant actions) allow to significantly improve the accuracy of the calculation.

As already mentioned in the introduction, our studies with twisted-fermions now are concerned $N_{F}=2$ twisted fermions, whereas the production of $N_{f}=2+1+1$ configurations is in progress [4]. In the $N_{F}=2$ setup, the strange quark is quenched as sea quark, implying an unknown uncertainty to our determinations of $f_{K}$ and $f_{K} / f_{\pi}$. We notice a posteriori, that however no systematic differences are observed in fig. 3 , where both $N_{f}=2$ and $N_{f}=2+1$ studies are shown. As far as the other systematics due to discretisation errors, finite size effects (FSE) and chiral extrapolation are concerned, our $N_{F}=2$ simulations have involved three values of the lattice spacing, $a \simeq 0.10 \mathrm{fm}, 0.09 \mathrm{fm}, 0.07 \mathrm{fm}$, two values of the lattice volumes $L=2.2$ and $2.9 \mathrm{fm}$ and pion masses as light as $260 \mathrm{MeV}$. In fig. 3 , we plot our $f_{K}$ and $f_{\pi}$ values at the simulated pion masses for all our values of $a$ s and $L s$. Data from different lattice spacings and volumes show small discrepancies (their size is consistent to $a^{2}$ scaling and ChPT-FSEs), proving that these systematics are under control in our study. 


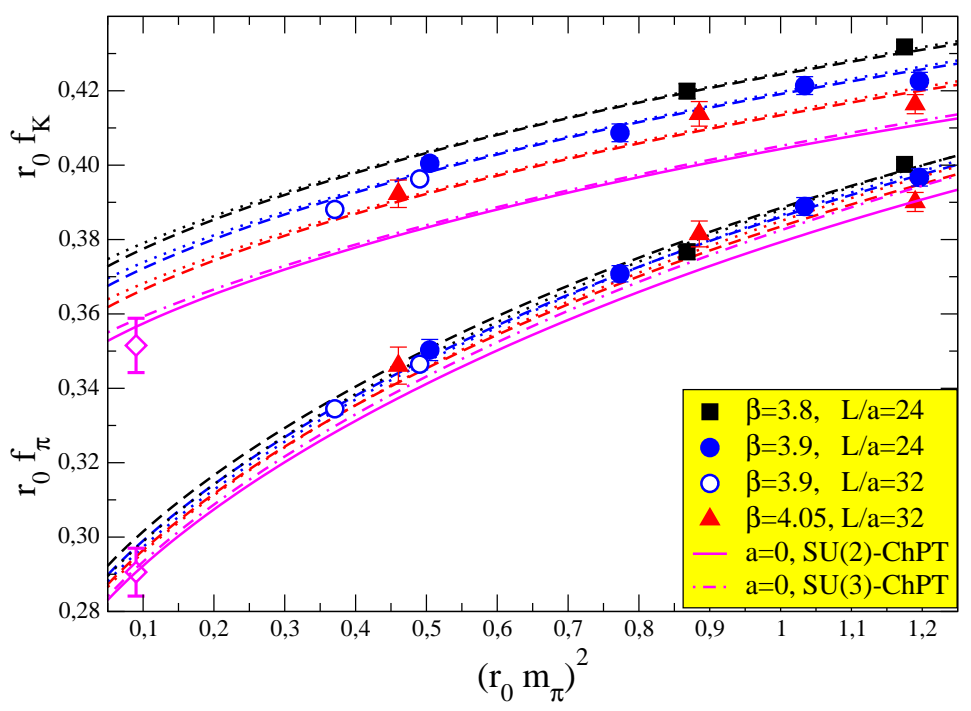

Figure 2: Our lattice results for $f_{\pi}$ and $f_{K}$ as a function of the simulated pion mass square. The SU(2)(SU(3)-) ChPT fit are also drawn.

In order to estimate the decays constants at the physical masses and in the continuum limit, the continuum and chiral extrapolations of the lattice results are performed simultaneously. More specifically, terms of $\mathscr{O}\left(a^{2}\right)$ and $\mathscr{O}\left(a^{2} \mu_{s}\right)$, coming from the Symanzik expansion of the lattice theory, are added to the ChPT expression. Moreover, both $\mathrm{SU}(2)$ and $\mathrm{SU}(3) \mathrm{ChPT}$ have been studied. The results are found in good agreement with each other, even though the SU(3)-ChPT fit looks less robust than the one based on $\mathrm{SU}(2)$.

Our final results [9] for the kaon decay constant and the ratio $f_{K} / f_{\pi}$ are then

$$
f_{K}=158.1(0.8)(2.3) \mathrm{MeV} \quad, \quad f_{K} / f_{\pi}=1.210(6)(17),
$$

where the first error comes from statistics and chiral extrapolation, the second from a conservative estimate of residual discretisation effects and FSE corrections. Our determination of $f_{K} / f_{\pi}$ leads to a determination of $\left|V_{u s}\right|$ that is in good agreement with the value obtained from semileptonic kaon decays, though with a larger error, as well as with the first row unitarity constraint of the CKM matrix.

\section{ETMC determination of $f_{+}(0)$ Form Factors}

$f_{+}(0)$ is the vector form factor at the zero momentum transfer $\left[q^{2}=\left(p_{K}-p_{\pi}\right)^{2}=0\right]$, defined by the $K \rightarrow \pi$ matrix elements $\left\langle\pi\left(p_{\pi}\right)\left|\bar{s} \gamma^{\mu} u\right| K\left(p_{K}\right)\right\rangle$.

Our determination of $f_{+}(0)$ with $N_{F}=2$ twisted-mass QCD fermions has been recently presented in ref. [6]. Following previous studies, we exploit the double-ratio strategy ${ }^{2}$ from [27, $28,29]$ to improve the $f_{+}(0)$ accuracy. As novelty to older works however, pion masses as light as $260 \mathrm{MeV}$ with the condition $M_{\pi} L \gtrsim 4$ have first been investigated. For the time being, our

\footnotetext{
${ }^{2}$ Here the $K \rightarrow \pi$ form factors are normalized to $K \rightarrow K$ and $\pi \rightarrow \pi$ ones and this allows to partially cancel different sources of uncertainties.
} 


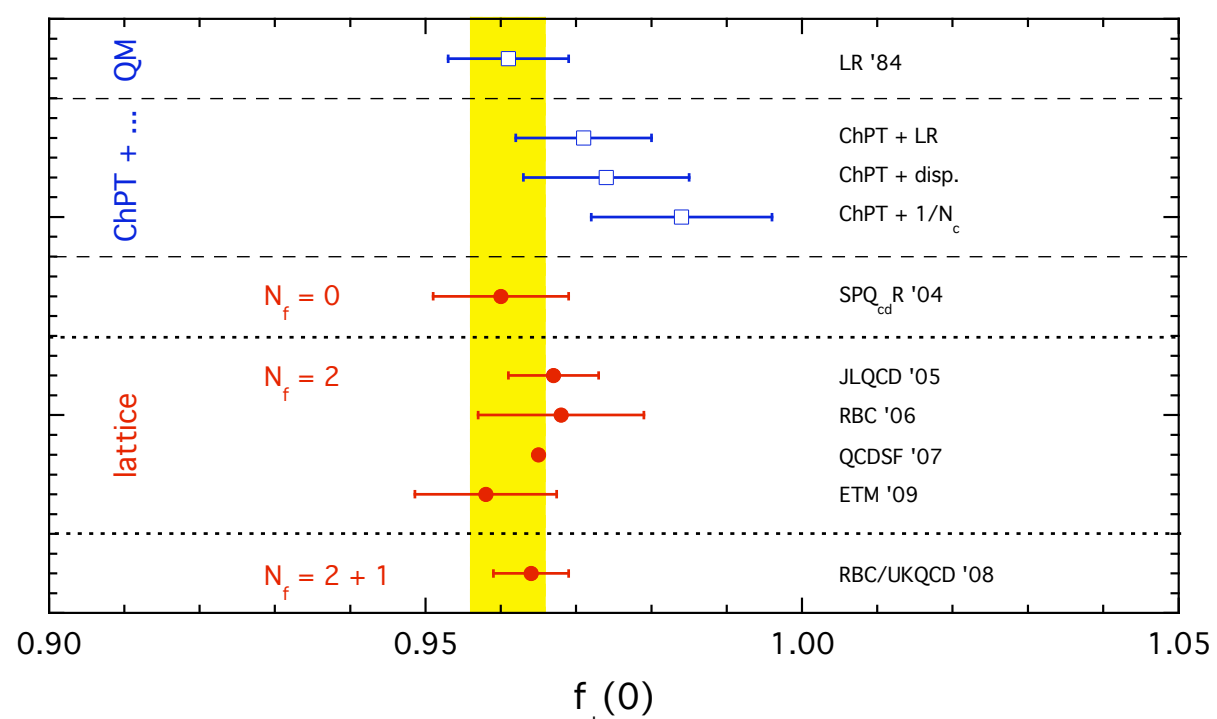

Figure 3: Lattice QCD $\stackrel{+}{D}$ determinations of $f_{+}(0)$.

simulations is only complete for one value of the lattice spacing, $a=0.009 \mathrm{fm}$ but further simulations at finer lattice spacing are in progress. To estimate discretisation effects, we use a sample of simulations at a finer lattice spacing, $a=0.007 \mathrm{fm}$ for $M_{\pi}=370$ and $M_{\pi}=470$.

As far as the chiral extrapolation of $f_{+}(0)$ is concerned, we have first investigated both $\mathrm{SU}(2)$ and SU(3) ChPT and like in the decay constants case both SU(2) or SU(3) fits lead to consistent results of $f_{+}(0)$. It is worth to stress that in the case of $f_{+}(0)$ the two strategies behave highly different, because of the conservation of vector current (CVC) $\mid \bar{s} \gamma^{\mu} u$ in the SU(3) limit. Shortly,

$$
\begin{aligned}
& f_{+}^{S U(3)}(0)=1+f_{2}+f_{4}+\mathscr{O}\left(M_{\pi, K} /\left(4 \pi f_{\pi}\right)\right)^{6} \\
& f_{+}^{S U(2)}(0)=F_{+}-\frac{3}{4}\left(M_{\pi} /\left(4 \pi f_{\pi}\right)\right)^{2} \log \left(M_{\pi}^{2}\right)+c_{+} M_{\pi}^{2}+\mathscr{O}\left(M_{\pi} /\left(4 \pi f_{\pi}\right)\right)^{4},
\end{aligned}
$$

In the $\mathrm{SU}(3)-\mathrm{ChPt}, f_{+}^{S U(3)}(0)=1$ reflects the $\mathrm{CVC}$ in the $\mathrm{SU}(3)$ limit and $f_{2}$ is unambiguisly predicted. Thus, the low-energy constants (LECs) appearing the NNLO $f_{4}$ correction. The task to determine $f_{+, 0}$ in the SU(3)-ChPT is thus reduced to the problem of finding a prediction for $f_{4}$. $\mathrm{SU}(2) \mathrm{ChPT}$ instead works in the framework $m_{\pi} \leq m_{K}$, and explicitly breaks the SU(3) limit. Then, LECs already appear at LO by $F_{+}$and at NLO by $c_{+}$. In order to determine $f_{+}(0)$ in this case, we fit these unknown coefficients from the lattice data. In ref. [6], we quote as final value of $f_{+}(0)$

$$
f_{+}(0)=0.9560 \pm 0.0057_{\text {stat. }} \pm 0.0062_{\text {syst. }} .
$$

In the systematic errors, we have included in quadrature chiral extrapolation, discretisation, finite size effects and the quenching the strange quark. To estimate the quenched effect, we exploited the fact that within SU(3) ChPT the difference between $N_{F}=2+1$ and $N_{F}=2$ setup is known at NLO, $f_{2}^{N_{F}=2+1}-f_{2}^{N_{F}=2}=-0.0058$.

Our determination agrees well with present $N_{F}=0 N_{F}=2$ and $N_{F}=2+1$ lattice calculations $[27,18,16,30]$ (see fig. 4) and using the latest determinations of $\left|V_{u d}\right|=0.97418(27)$ and $\left|V_{u s}\right| f_{+}(0)=0.21668(45)$, we get from (4.3) $\left|V_{u d}\right|^{2}+\left|V_{u s}\right|^{2}+\left|V_{u b}\right|^{2}=1.0004 \pm 0.0015$. 


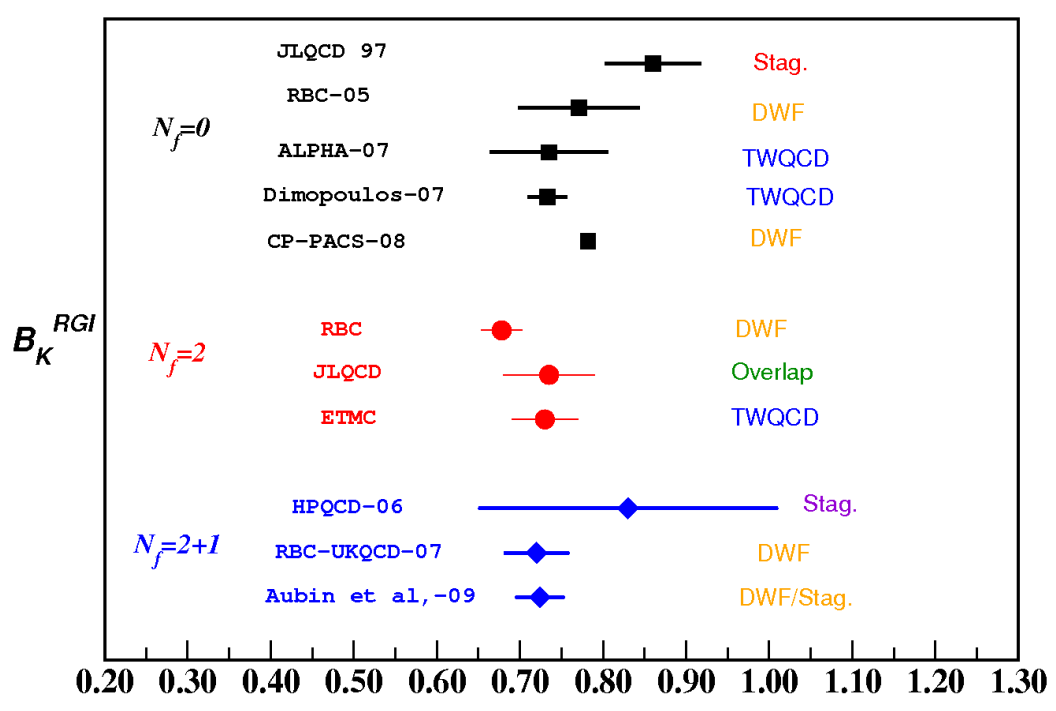

Figure 4: Lattice QCD determinations of $B_{K}^{R G I}$.

\section{ETMC Determination of the $B_{K}$ parameter}

As final quantity, I will now summarize our calculation of the bag parameter $B_{K}, B_{K} \propto$ $\left\langle K^{0}\left|\bar{s} \gamma_{L}^{\mu} d \bar{s} \gamma_{L}^{\mu} d\right| \bar{K}^{0}\right\rangle$, with $N_{F}=2$ maximally twisted-mass fermions. The ETMC study of ref. [31] has been now finalized, including simulations at finer lattice spacing $(a=0.007 \mathrm{fm})$ and a combined chiral and continuum fit (with $M_{\pi} \gtrsim 260 \mathrm{MeV}$ ). Our preliminary value [32] is $B^{R G I}=0.73(42)$, and is shown together with other estimates in fig. 5. Our value is in good agreement with other unquenched estimates and importantly is as precise as determinations from chiral-invariant fermions. This is definitely a merit of twisted-mass fermions [33] with respect to older Wilson-like simulations.

In the case of Wilson or Clover fermions for example, where the Wilson terms in eq. (2.1) is a singlet in the flavour sector (e.g. $r_{f}=1 \vee f$ ), chiral symmetry is badly broken and the operator $Q^{\Delta S=2}=\bar{s} \gamma_{L}^{\mu} d \bar{s} \gamma_{L}^{\mu} d$ related to $B_{K}$ inevitably mixes with wrong-chirality contributions. In principle, one can try to evaluate this wrong-chirality mixing and over the years different techniques such as RI-mom renormalisation and/or appropriate Ward-Identities have been introduced. However, as finally we learn from these studies, $B_{K}$ determinations with Wilson and Clover formulations turn out to be quite poor [34]. The practical difficulty is not simply the evaluation of the mixing coefficients but the necessity to subtract them with a high level of accuracy.

In the twisted-mass formulation of Wilson fermions instead, the $r_{f}$ Wilson parameter can be set differently for any flavour species. Now by a suitable combinations [35] of $r_{f}$ signs for each (valence) quark fields of $Q^{\Delta S=2}(\mu)$ we can ensure multiplicative renormalisation (no mixing) as well as automatic $\mathscr{O}(a)$ improvement.

In the specific case of our study, we have set the first pair of $s$ and $d$ quarks in $Q^{\Delta S=2}$ in the combination $r_{s}=r_{d}$ and the latters with $r_{s}=-r_{d}$. The multiplicative renormalisation is performed in the RI/MOM scheme and the absence of mixing with wrong chirality operators is explicitly shown in fig. 5, where the mixing coefficient of $\bar{s} \gamma_{\mu_{R}} d \bar{s} \gamma_{R}^{\mu} d$, with $Q^{\Delta S=2}$ is shown. 


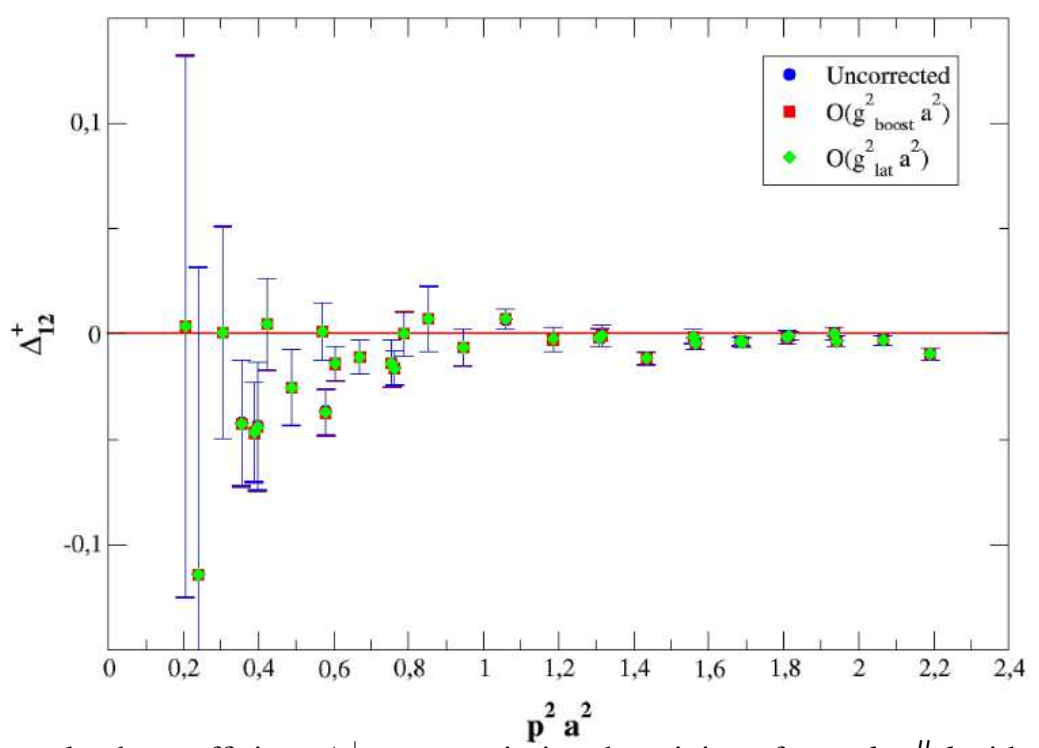

Figure 5: As example, the coefficient, $\Delta_{12}^{+}$, parametiseing the mixing of $\bar{s} \gamma_{\mu_{R}} d \bar{s} \gamma_{R}^{\mu} d$ with $Q^{\Delta S=2}$ is shown. The relative value is compatible with zero.

\section{Conclusions}

In this write-up, lattice estimates of $f_{+}(0), f_{K} / f_{\pi}$ and $B_{K}$ with maximally twisted-mass fermions by ETMC has been presented. Our determinations are compatible with current unquenched estimates, confirming that twisted-mass fermions are a valid lattice approach. It is in turn computationally cheap. These studies are still for $N_{f}=2$ simulations. The good agreement observed among the recent $N_{f}=2$ and $N_{f}=2+1$ lattice determinations suggests, however, that such an effect is smaller than the other systematic uncertainties estimated.

\section{References}

[1] Ph. Boucaud et al. [ETMC], Phys. Lett. B 650, 304 (2007) [arXiv:hep-lat/0701012]; Comput. Phys. Commun. 179, 695 (2008) [arXiv:0803.0224 [hep-lat]].

[2] R. Frezzotti, P.A. Grassi, S. Sint and P. Weisz, JHEP08 (2001) 058 [hep-lat/0101001].

[3] R. Frezzotti and G.C. Rossi, JHEP08 (2004) 007 [hep-lat/0306014].

[4] R. Baron et al. [ETMC], PoS LATTICE2008 (2008) 094 [arXiv:0810.3807 [hep-lat]].

[5] M. Antonelli et al. [FlaviaNet Working Group on Kaon Decays], arXiv:0801.1817 [hep-ph].

[6] V. Lubicz, F. Mescia, S. Simula, C. Tarantino and f. t. E. Collaboration, arXiv:0906.4728 [hep-lat].

[7] V. Cirigliano, M. Gonzalez-Alonso and J. Jenkins, arXiv:0908.1754 [hep-ph].

[8] G. Isidori and P. Paradisi, Phys. Lett. B 639, 499 (2006) [arXiv:hep-ph/0605012]; W. S. Hou, Phys. Rev. D 48, 2342 (1993).

[9] B. Blossier et al., [ETMC] JHEP 0907, 043 (2009) [arXiv:0904.0954 [hep-lat]].

[10] V. Bernard, M. Oertel, E. Passemar and J. Stern, JHEP 0801, 015 (2008) [arXiv:0707.4194 [hep-ph]]. 
[11] A. J. Buras and D. Guadagnoli, Phys. Rev. D 79, 053010 (2009) [arXiv:0901.2056 [hep-ph]]; Phys. Rev. D 78, 033005 (2008) [arXiv:0805.3887 [hep-ph]].

[12] E. Lunghi and A. Soni, JHEP 0908, 051 (2009) [arXiv:0903.5059 [hep-ph]]; Phys. Lett. B 666, 162 (2008) [arXiv:0803.4340 [hep-ph]].

[13] C. Allton et al. [RBC-UKQCD], Phys. Rev. D 78, 114509 (2008) [arXiv:0804.0473 [hep-lat]].

[14] S. Durr et al., [BMW] Science 322, 1224 (2008) [arXiv:0906.3599 [hep-lat]].

[15] L. Del Debbio, L. Giusti, M. Luscher, R. Petronzio and N. Tantalo, JHEP 0702, 082 (2007) [arXiv:hep-lat/0701009].

[16] D. Brommel et al. [QCDSF], PoS LAT2007, 364 (2007) [arXiv:0710.2100 [hep-lat]].

[17] S. Aoki et al. [PACS-CS], arXiv:0807.1661 [hep-lat].

[18] S. Aoki et al. [JLQCD and TWQCD], Phys. Lett. B 665, 294 (2008) [arXiv:0710.1130 [hep-lat]].

[19] C. Aubin, J. Laiho and R. S. Van de Water, arXiv:0905.3947 [hep-lat].

[20] K. Jansen, arXiv:0810.5634 [hep-lat]; L. Lellouch, arXiv:0902.4545 [hep-lat].

[21] ETMC, “Light Meson Physics from Maximally Twisted Mass Lattice QCD,” in preparation; arXiv:0810.2873 [hep-lat]; K. Jansen, M. Papinutto, A. Shindler, C. Urbach and I. Wetzorke [XLF], JHEP 0509, 071 (2005) [arXiv:hep-lat/0507010]; D. Becirevic et al., Phys. Rev. D 74, 034501 (2006) [arXiv:hep-lat/0605006].

[22] Y. Aoki et al., Phys. Rev. D 72 (2005) 114505 [hep-lat/0411006].

[23] C. Aubin et al. [MILC], Phys. Rev. D 70 (2004) 114501 [hep-lat/0407028]; C. Bernard et al., PoS LAT2007 (2007) 090 [arXiv:0710.1118 [hep-lat]].

[24] E. Follana, C. T. H. Davies, G. P. Lepage and J. Shigemitsu [HPQCD/UKQCD], Phys. Rev. Lett. 100, 062002 (2008) [arXiv:0706.1726 [hep-lat]].

[25] S. Aoki et al. [PACS-CS], 0807.1661 [hep-lat].

[26] C. Aubin, J. Laiho and R. S. Van de Water, 0810.4328 [hep-lat].

[27] D. Becirevic et al., Nucl. Phys. B 705, 339 (2005) [arXiv:hep-ph/0403217].

[28] S. Hashimoto et al., Phys. Rev. D 61, 014502 (1999) [arXiv:hep-ph/9906376].

[29] P. A. Boyle, J. M. Flynn, A. Juttner, C. T. Sachrajda and J. M. Zanotti, JHEP 0705, 016 (2007) [arXiv:hep-lat/0703005]; D. Becirevic, B. Haas and F. Mescia, PoS LAT2007, 355 (2007) [arXiv:0710.1741 [hep-lat]].

[30] P. A. Boyle et al., Phys. Rev. Lett. 100, 141601 (2008) [arXiv:0710.5136 [hep-lat]].

[31] P. Dimopoulos et al., PoS LATTICE2008, 271 (2008) [arXiv:0810.2443 [hep-lat]].

[32] S. Simula talk and proceedings at Lattice 2009.

[33] P. Dimopoulos at al., Nucl. Phys.B749 (2006) 69 [hep-ph/0601002]; C. Pena, S. Sint and A.Vladikas, JHEP09 (2004)069 [hep-lat/0405028]; P. Dimopoulos, H. Simma and A. Vladikas, JHEP 0907, 007 (2009) [arXiv:0902.1074 [hep-lat]].

[34] D. Becirevic et al. Eur. Phys. J. C 37, 315 (2004) [arXiv:hep-lat/0407004].

[35] R. Frezzotti and G.C. Rossi, JHEP10 (2004) 070 [hep-lat/0407002]. 